\title{
Barriers to Cervical Screening among Pacific Women in a New Zealand Urban Population
}

\author{
Sunia Foliaki*, Anna Matheson
}

\begin{abstract}
Background: In Aotearoa/New Zealand cervical screening programmes have reduced cervical cancer; however, half of cervical cancer cases among Pacific women are found among clients who had not attended cervical screening. Hence, we set out to determine health provider perspectives on barriers that prevent their services reaching Pacific women within Aotearoa/New Zealand. Materials and Methods: Twenty semi-structured interviews were conducted with health care providers, Pap smear takers and community workers in the Wellington region.Participants were asked their views on factors that enabled and/or constrained the participation of Pacific women in their cervical screening services. Results: Six interrelated themes influencing participation in cervical screening among Pacific women in the Wellington region were apparent: the funding and practice of service delivery; family always coming first; the cost of screening services; type of employment; the appropriateness of information; and attitudes to self and screening. Conclusions: Determining specific ethnic group actual health needs and meeting them contributes to overall improvement in New Zealand's health status. The results identified the need for improvements to the delivery of screening services including adapting cervical screening services to the requirements of Pacific women through more outreach services at alternate clinic hours; culturally appropriate practitioners; the ability to take up opportunities for health checks and foster long-term relationships; as well as appropriate monitoring and evaluation of approaches. Funding and reporting relationships also need to be compatible with the goal of improving outcomes for Pacific women. Further research into client voices for their particular needs to compliment the service provider perspective as well as minority groups is called for.
\end{abstract}

Keywords: Cervical cancer - screening - pacific women - Aotearoa/New Zealand

Asian Pac J Cancer Prev, 16 (4), 1565-1570

\section{Introduction}

The Pacific population generally in New Zealand grew from the recent immigrant community of the 1940's of just over 2000 to over a quarter of a million in 2013 and now make up seven per cent of the total population and the fourth largest ethnic group. The leading three ethnic groups being Europeans (74\%), Maori (15\%) and Asian $(12 \%)$ whose population size almost doubling between 2001 and 2013. The Pacific population in New Zealand are often referred to as Pasifikans or Pacifica and generally refer to populations originating from and identifying with common geographic origins from the Pacific islands (including Samoa, Tonga, Cook Islands, Fiji, Niue, Tokelau, Kiribati and Tuvalu), a shared sense of common origins and ancestry and sharing a common culture, customs or language. A diverse ethnic group, this sub population accounts for a disproportionately significant share of health burdens compared to most other N.Z. population groups and the general population-except Maori-across a number of health outcomes including a lower life expectancy and higher morbidity and mortality rates of chronic diseases (Minister of Health and Minister of Pacific Island Affairs, 2010), including certain easily preventable cancers. Cervical cancer is the third most common cancer in women globally with more than $85 \%$ of this burden occurring in developing countries. In parts of the Pacific (Melanesia) cervical cancer remains the most common cancer among women and is the third leading cancer among women in Tonga, the Cook Islands, Niue and among Pacific women in Aotearoa/New Zealand (N.Z.). Compared to the Pacific region, cervical cancer rates are low for the general population in N.Z. However, Pacific peoples and the indigenous Maori experience a disproportionately higher rate of certain cancers and in particular, infectious related cancers such as cancer of the cervix (Foliaki et al., 2011; Obel et al., 2014) and liver (Foliaki et al., 2004; Meredith et al., 2012). Pacific women also have higher rates of other gynaecological cancers (endometrial, ovarian) (Meredith et al., 2012).

Pap smear screening and its effectiveness in reducing the incidence and mortality from cervical cancer has been well documented (Taylor et al., 1996; Mehmetoglu et al., 2010) including studies involving Pacific people (Chen and Koh, 1997) overseas and in N.Z. (Cox and Skegg, 1992). The N.Z. National Cervical Screening Programme was 
established in 1990. However, the uptake of Pap smears is not the same across population groups. For example Pacific women have been shown to have low cervical screening rates 7 resulting in the failure to detect cervical abnormalities early before they become cancerous. Pacific women have a lower uptake of cervical screening with just over half of eligible Pacific women having regular cervical screening (http://www.nsu.govt.nz/news/3696. aspx. 2013) compared to over seventy percent among the total population and over eighty percent among pakeha/ palangi women. The cost of a cervical smear test is what one normally pays to see one's doctor or nurse although some community or primary health organisations offer a free or low-cost service.

From the existing literature on why screening services do not meet the needs of certain population groups some of thebarriers reported include, language difficulty, in a review of the literature pertaining to cervical cancer and Pap testing among Asian populations in North America (Taylor et al., 1996) as well among both Asian and Pacific islanders in North America (Dang et al., 2010) and feelings of shyness and embarrassment were reported among Maori and Pacific women for whom exposing bodies in the process of smear taking compromises cultural beliefs about sacredness (Lovell et al., 2007), and an anxiety about lack of confidentiality within small community groups. A reluctance to discuss topics related to sexuality or the reproductive organs was reported in a study among Pacific women in New Zealand (Sligo et al., 1998) and a perceived relationship between cervical smears and sexual activity as reported in assessment of attitudes among Pacific women to cervical screening in a New Zealand central region (Jameson et al., 1999; Lovell et al., 2007) are also among related issues frequently reported. There is very limited research published, however, that looks specifically at Pacific women within N.Z. and why they are not accessing screening services. As a step in addressing this research gap, our study has set out to interview providers of screening services to gather their perspectives on why their services are not reaching Pacific women. The research findings provide direction for improving the delivery of screening services in N.Z. as well as for guiding further research and investigation into improving participation among Pacific women in cervical screening activities. The research also has implications for other ethnic minority groups and sub-populations with high cervical cancer morbidity and mortality rates linked to low cervical screening-including Maori.

\section{Materials and Methods}

Twenty face to face, semi-structured interviews were carried out with service providers to explore what enables and constrains effective cervical screening services to Pacific women in Wellington, N.Z. A purposive, snowballing sampling strategy was used to select participants as the aim was to gather interviews that would be 'data-rich' in the topic area. Some broad selection criteria included health care services providing Pacific health primary care services in areas with significant Pacific populations, public health and primary health organization targeting
Pacific island and low income population and Pacific nursing institutions in the Wellington who are involved in cervical screening. The interviewees are current employees of organizations and health services that provide primary health services to over $80 \%$ of Pacific populations in the Wellington region. by organizations that All the interviews were in English and conducted face-to- face by the principal investigator at a time and place chosen by the participant. As each interview was undertaken the participant was asked to suggest another appropriate participant for interview. The sample size in the study was determined when no new individuals were identified for interview. Each interview was digitally recorded and transcribed verbatim. Each transcript was coded by hand using a content analysis approach independently by two researchers. The two investigators then came together to discuss, compare and agree on the themes that had arisen through the coding. Transcripts were uploaded into the qualitative software NVivo and each was coded according to the identified themes. The themes were then grouped and again the two investigators came together to determine the relevance of the data grouped by theme.

The interviewees consisted of nineteen women and one man who were recruited with assistance from Pacific health service providers and through other community networks including the Porirua Union and Community Health Service and the Pacific Peoples Health Advisory and Hutt Valley District Health Board. The majority of participants were Pasifikans-eight of whom were primary health care nurses and qualified Pap smear takers working with service providers with high numbers of Pacific clients. Other participants included nursing school tutors involved in Pacific health training, public health administrators, nursing students and community workers. The majority of the participants identifying as Pasifikans were first generation immigrants and there were two pakeha/palangi primary health care nurses. Pakeha and palangi originally referred to early European settlers in N.Z. but recently generally referring to 'white' nonMaori or non-Polynesian people. This research has been evaluated by the Ministry of Health's Health and Disability Ethics Committee and judged to be low risk. Written informed consent was obtained from all participants.

\section{Results}

Six interrelated themes emerged from the analysis. These were: the funding and practice of service delivery; family always coming first; the impacts of cost of screening services; type of employment; the appropriateness of information and attitudes to self and screening.

\section{The funding and practice of service delivery}

The way that services were run was reported by participants to impact on the delivery and uptake of screening by Pacific women. Issues discussed included; a difficult funding environment for service providers with both limited resources and frequent changes to the funding rules; a lack of appropriately skilled practitioners; few Pacific practitioners; and an identified need for flexibility within the practice to be able to be opportunistic when and 
where to screen Pacific women.

The training of more Pacific health practitioners was viewed as critical to the effectiveness of screening services, but all participants regarded the current numbers as well below what would be desirable. Greater resources for outreach services-which gave providers not only access to clients but also an appreciation of their economic, social and domestic situations-was also identified as critical. They walk in, there would be a child that's sitting somewhere...friends' and relative's kids too, elderly, disabled partners...and they look in the house oh it's all torn here or it's quite dirty or is it the hygiene that's the problem? Is it because it's a leaky home? (Int 24: Outreach nurse and Pap smear taker)

The days of sitting in the office and letting them come to you is no longer there.... take the service out to them, find out what their needs are first and work along with them. (Int 22: Regional cervical screening service nurse)

The importance of skilled practitioners was raised frequently. Particularly the impact on women not returning for smears if a screening session was not a positive experience for them. So it is actually important to have skilled practitioners, one bad smear experience will affect far more than just that individual woman. (Int 25: Community health service nurse and Pap smear taker)

There was also a shared view that there were too few Pasifikan smear takers. ...it is very tight now...we're not able to employ another nurse. Even though we could do with another nurse...(Int 20: Primary care nurse and cervical smear nurse).

One participant argued that this issue of representation has not been addressed throughout her two decades of working in Pacific health. Similarly it was expressed that Pacific "sections" established within health organisationsboth official and semi-official-were often disbanded despite Pacific workers advocacy for more Pacific voice at the policy level. Others noted that there were existing opportunity for Pacific voices to be heard (through consultation processes), however, these perspectives were not adequately heeded. ... and I've seen this trend go up and out, go up and out, go up and out. And when you are fighting (and) advocating for the rights of what you believe in and to make a difference for our Pacific people and for priority people and you get pushed back and pushed back. It can be a lonely road sometimes...(Int 22: Regional cervical screening service nurse)

One participant further suggested their own service policies and practices were not realistic and not client oriented in order to get the best outcomes for the populations they serve.... the policy at the moment is you contact three times and that's it, "three strike (sic) you're out". You ring, you send a letter and or visit and if they don't turn up that's it. .. That's what. (Int 38: Primary Health Organization nurse)

Rather, it was strongly viewed by participants that outreach services, including home visits and having flexibility in how contact was made with women, was the way forward. For example it was believed that health staff could be more vigilant and actively enquire for updates on other issues from women, like vaccination coverage and Pap smear when they attended for other health issues.
The potential benefits of outreach services were clearly articulated, but so to were the limited structural supports for this kind of activity. ... if the Plunket (mother and child support organisation) services provide up to six weeks home visits why can't mothers have their Pap smear at six weeks at home too during the final weeks of Plunket visits... (Int 31: Nursing school tutor (Pacific health programme))

Participants saw numerous benefits in outreach services for smear taking including the information such visits provide on the wider living circumstances of women not coming into clinics for smears. For example having children or elderly at home, housing circumstance and location as well as church and religious affiliations. The extension of clinic hours into the evenings on occasion when workers return home was viewed as one useful approach to accessing women.

\section{Putting family first}

The family 'role' that Pacific women take on was identified as impacting on their ability to prioritise taking time for a smear. Family priorities included caring for dependents and working to earn income for the family. My husband, my kids, my family, my in-laws will be before me. (Int. 23: Primary Health Organization Outreach nurse)

It was a common view held amongst participants that women in Pacific communities are the overall operational managers of the household-overseeing child minding, health care, care of the elderly and disabled, income earner as well as nurturer of the family. This role often means a woman's personal health concerns are seen as a low priority-attending to family needs before their own. Rather than women avoiding participating in screening, it is more accurate to view them as prioritising total family care at the expense of their personal care such as a Pap smear. For instance an appointment for a Pap smear would rarely take precedence over looking after grandchildren after school. ... you live for your family, you don't live for yourself and Pap smears are very much an individual care... as the nurturers and managers of the household looking after your own health is way down the list...you are raising children, you are getting people fed, running the household, you are paying the bills... (Int 35: Regional Public Health Advisor)

Participants identified that their clients often had multiple jobs in order to earn an adequate income to meet family needs. Prevention measures such as screening were a low health priority.

\section{Direct and indirect costs of attending screening}

A dominant theme to emerge from the interviews was that of cost. It was argued that barriers were created for Pacific women through both the direct and indirect financial costs of participating in cervical screening. These included the costs of attending a Pap smear but also the opportunity costs in terms of family, work and income. Also highlighted was the inconsistent information provided about the direct costs of screening to women.

All participants identified the cost of Pap smears as a major barrier to Pacific women attending cervical screening. The cost for a Pap smear is almost always weighed against more immediate household needs such 
as food items. I rang a few doctors and the cost was from zero to $\$ 75$ for a smear. I'm not going to actually waste the $\$ 75$ on me (Int 39: Health Promotion Coordinator (Pacific health)) ... and even...if it's going to cost $\$ 40$ forget it, I'd rather have that $\$ 40$ to buy milk or bread for my family... (Int 22: Regional cervical screening service nurse).

A number of participants felt even where Pap smear services were either free or practically free there were still significant indirect costs (transport, time, childcare) that impacted on those on low incomes. Interestingly, not all providers appear to make it widely known they offer free smears. According to some participants women were not always aware of certain services providing smears free. Indeed, because of financial pressures on service providers themselves, their own 'codes of practice' may ...not actively let women know that smears have been met by a $P H O$...(Int 22: Regional cervical screening service nurse).

\section{Employment circumstance}

All participants suggested that the type of work prevalent among Pacific women in the region was not conducive to having appointments during working hours. It was suggested that expectations within the work environments (of factories or for cleaners for example) make it difficult to postpone work to attend appointments for what is not seen as illness. Even if work encouraged screening appointments the prevalence of Pacific workers juggling between changing shift work often meant that clinic hours coincided with shift workers resting time Timing yeah, if I go to a practice who actually works from eight to five and I hold three jobs because my children needs, I actually have to look after my family, my first priority is to my family, that they're taken care of (Int 39: Health Promotion Coordinator (Pacific health))

\section{Information on screening}

The majority of participants suggested that there was need for more and better information on Pap smears for Pacific women. Conveying health messages was argued to be more effective if it were through personal contacts as opposed to written information and general health messages. Again, outreach services were suggested as an appropriate vehicle for giving meaningful, relevant information to women. Within the community this translated to involvement in health promotion within churches and other community groups. I don't think that just putting a few ads on TV and having posters sprinkled around in doctors surgeries are enough. We need to look at ways to engage churches and community groups in an ethnic specific way. (Int 35: Regional Public Health Advisor).Greater evaluation of current attempts at engaging women in screening was seen as urgently needed. It was suggested that providers often continued with similar approaches even when they have no information on whether they have worked in the past ...health education materials...continue to be delivered year after year without any kind of review (Int 35: Regional Public Health Advisor)...

Client perspective

Participants' felt that Pacific women's attitudes towards cervical screening were a combination of cultural, family, social and economic factors. These included concerns for confidentiality when examined by someone known to them because of being part of a close community where social standing and respect are integral. ... see I think that respect part of our cultural that you know it's another sister, it's another mother that's going to do those part,... that part. You know if it was to deal with a dressing oh yeah come and do it. ..even some older women come and said oh I don't want you to do it, yeah it's not respectful that you have to do this... It's really the respect that these people have for you... even worse when they found out that I was a pastor's wife! (Int 24: Outreach nurse and Pap smear taker).

Cultural views about a woman's body and sexuality were seen as an important driver of women's attitudes to screening. One participant for example stressed that at a very young age Pacific girls are given the message that, It is not an acceptable area (sexuality) for open discussion... Pap smears are in some way has a connection with that... (Int 31: Nursing school tutor (Pacific health programme))

It was suggested that older clients may struggle with the connection between preventive medicine and long term health outcomes. This was illustrated by a frequent suggestion that once child bearing is over the reproductive organs are not a priority or at risk anymore. ... I've had my children so nobody getting down there and have a look you know, it's closed door down there. (Int 22: Regional cervical screening service nurse).

Participants felt that it was also difficult to emphasise the importance of preventive measures more generally. It was suggested that women felt there was no point attending appointments if they did not feel sick and worse still, there was often a feeling that having a smear was "looking" for cancer and one may end up finding cancer although one feels perfectly well.... why fix something if they don't feel anything wrong And you know even big people will say well why should we do things when we are still healthy (Int 24: Outreach nurse and Pap smear taker) .. Imean a Pap smear you're looking for trouble (cancer). Before they go they feel alright, once they have been to the doctor, they are told oh no you have got cancer,... they don't want to know (Int 25: Community health service nurse and Pap smear taker).

\section{Service delivery attitude}

Staff attitudes were identified as contributing to Pacific women's active participation in cervical services as well as other services provided at primary care level. It was viewed as necessary that practitioners have an appreciation of minority cultures; that their outlook on health seeking behaviour should acknowledge community perspectives as well as those of individuals. It was frequently suggested that practitioners needed to realise that the main goal not just to get quick return by achieving a single Pap smear, but also paving the way for long term relationship facilitating future Pap smears. ..the first time the nurse is rude, they won't come back, the receptionist is rude, they won't come back, being embarrassed by something they won't come back. (Int23: Primary Health Organization Outreach nurse). We as nurses don't mind it, ... I don't 
mind it if somebody says I don't want this Indian doing my smear or because she lives down the street, that's fine I understand...will try to get some other nurses because there is always another nurse, you can do it. (Int23: Primary Health Organization Outreach nurse).

All participants expressed a need for being mindful of the longer term. For example if a client refuses a Pap smear this year you have a better chance of getting a Pap smear the next year by respecting their refusal. Establishing rapport in the long-term also opens up the opportunity to address other health issues. You can't go against their wishes for this is not the only smear that you will want from them because every three years they'll have to come back (so you want to have that open door policy of okay we can facilitate something else for you. (Int23: Primary Health Organization Outreach nurse)

\section{Discussion}

The main findings of this study resonate with previous research on the up-take of cervical screening, however, the research also highlights there is a gap in literature focusing explicitly on Pacific women within N.Z. Our research suggests that barriers to Pacific women taking up cervical screening are multi-level and they interact. In particular we argue that the 'cultural' role of Pacific women within their families and communities interacts with their socioeconomic circumstances as well as the resourcing and delivery modes of preventive health services such as screening.

One rare piece of work from 2001 to 2002, by Lovell and colleagues, targeted cervical screening in South Auckland which has a high Pacific population (Lovell et al., 2007). Their findings were similar to this research in that both socioeconomic factors as well as cultural beliefs around one's body were argued to impact on a woman's decision to participate in cervical screening. Whereas Lovell's research discussed attitudes relating to the degree to which cultures-and individuals within them-hold a 'medicalised' view of one's body, our research found participants frequently discussed a significant attitudinal response from clients to 'prevention' in terms of health. Participants felt that prevention for these women was not seen as a high priority given the family, cultural and socioeconomic contexts in which they lived. We found that some of these very practical 'cultural' and socioeconomic features included priority being given to the health and welfare of children and wider family as well as household economic needs being seen as more important than personal health prevention. As such the current delivery of cervical screening to Pacific women is perhaps not compatible with the priorities of Pacific families.

Although we identified barriers for Pacific women in prioritising preventive health care, other research shows that Pacific populations in N.Z. also do not access General Practitioners (GPs) at the same rates as pakeha/palangieven where it might be for acute health issues rather than prevention (Ludeke et al., 2012). The authors highlight features of the way GPs services are run that have potential to impact on the compatibility with Pacific clients. Among these barriers were: language and communication difficulties, consultations being perceived as rushed; a lack of flexibility in appointment times; how clients are 'received' at service provider receptions; and the need for greater Pacific presence were argued to impact on one's ability to access primary care. Cummings et al (Cumming, Mays, and Gribben 2008) further argue that because of these challenges there may be a need for greater nurse or other provider involvement in consultations rather than primarily involving doctors only.

These findings concur with our study in that there was a call for a greater and more appropriate Pacific workforce engaged in screening which could respond to language, communication and cultural factors in an appropriate way. Most prominent was the importance of flexibility in delivery, in terms of when and where Pacific women could be screened. Home screening was said to be discouraged or at least not encouraged among outreach nurses of some service provider's which may reflect the time and resource saving of 'managed care'. As captured in our interviews this situation led some nurses to bend the rules and take extra kits to individual homes and do Pap smears on a 'batch' of neighbouring women. It was also evident from our research that non-routine pap smears were carried out opportunistically to coincide with women gathering for community events. Similar to other research (Cory et al., 1995) we also found that greater monitoring and evaluation of health promotion and education materials was desired as evaluation of existing materials and programmes has been neglected.

One of the more common features discussed in the health services literature is the need for good patient/ service provider rapport (Ludeke et al., 2012). Less commonly discussed, however, is the need for developing a long-term relationship with women. For example a refusal by a woman for a Pap smear is still an opportunityif handled appropriately-to follow up at some point in the future.

The ability for screening services to be flexible and more responsive to the needs of Pacific women is influenced by the ways in which they are resourced and supported. Cervical screening services for women in N.Z. are incorporated into the existing delivery of primary care. The difficulties experienced and the changing nature of the funding structures for cervical screening services share challenges with the primary care sector as a whole and suffer the same difficulties in achieving equitable access for certain population groups. Influencing this situation has been the almost continual restructuring of N.Z's health care system over the last three decades. The hospital board model based on separate funding, planning and service delivery arrangements for hospitals, primary and public health care gradually merged district public health services with hospital boards creating Area Health Boards during the early to late 1980's. Sweeping changes resulted in establishment of Regional Health Authorities in the 1990's and then in 2000/2001 to District Health Boards (DHBs). These changes were all accompanied by changes to funding, purchasing and service provider arrangements including screening and public health, and community services (Cumming and Mays, 2002; Cumming et al., 2008). Indeed Lovell and colleagues 
(Lovell et al., 2007) argue that the major contributor to the women they interviewed being overdue for a smear was the structure of the health care system itself. Therefore, it is not surprising that the findings of our study included frequently expressed frustrations with difficulties in collaborating and coordinating programmes whose funding sources were impacted, and distracted, by a health system that is continually changing.

Moreover, research (Sheridan et al., 2011) exploring health equity within the N.Z. health care system found that despite substantial rhetoric for 'addressing equity' for hard-to-reach populations, the implementation and monitoring of health programmes was inadequate in terms of equity. For example, the authors argue that a disproportionate burden of care for reducing inequities is placed on nurse and community workers, but that it was not a condition of their employment that they have training in diversity and culture (Sheridan et al., 2011) . This situation perhaps compounds the problem identified in our research-that there are too few Pacific smear-takers. Sheridan and colleagues also identified that unlike Māorithe indigenous population of N.Z.-equity for communities defined by geographic area, or for other population groups-including Pacific people-is not embedded within government policy.

In conclusion, the Pacific populations in New Zealand have a disproportionate burden of non communicable diseases compared to most other N.Z. population groups.. These include cancers that are highly amenable to early detection and prevention such as cervical cancer. The compatibility of Pacific women's priorities and the delivery of screening services need to be addressed if mortality rates from cervical cancer are to be reduced for Pacific women. To achieve this compatibility, compelling Pacific women to change or disregard their inherent priorities seems impractical and unlikely to achieve success. More promising would be for services to be responsive to the needs and circumstance of Pacific women recognizing the direct and indirect barriers. From this research some of the solutions to adapting screening services to the needs of Pacific women include: offering outreach services, home and church visits; alternate clinic times; temporary care for dependents; culturally appropriate practitioners; the ability to take up opportunities for health checks and foster long-term relationships; as well as appropriate monitoring and evaluation of approaches used. For services to be responsive, however, their funding and reporting relationships also need to be compatible with the goal of improving outcomes for Pacific women. This research suggests that currently they are not. It would be illuminating to explore the experience of screening providers for Pacific women and Pacific women clients from other geographic areas within N.Z. as well as to investigate more closely the potential mechanisms for achieving more responsive services.

\section{References}

Chen MS Jr, Koh HK (1997). The need for cancer prevention and control among Asian Americans and Pacific Islanders. Asian Am Pac Isl J Health, 5, 3-6.
Cory J, Bottum C, Haddock C (1995). Evaluating print health education materials. Cancer Pract, 3, 54-6.

Cox B, Skegg DC (1992). Projections of cervical cancer mortality and incidence in New Zealand: the possible impact of screening. J Epidemiol Community Health, 46, 373-7.

Cumming J, Mays N (2002). Reform and counter reform: how sustainable is New Zealand's latest health system restructuring? J Health Serv Res Policy, 7, 46-55.

Cumming J, Mays N, Gribben B (2008). Reforming primary health care: is New Zealand's primary health care strategy achieving its early goals? Aust New Zealand Health Policy, 5,24 .

Dang J, Lee J, Tran JH, et al (2010). The role of medical interpretation on breast and cervical cancer screening among Asian American and pacific islander women. J Cancer Educat, 25, 253-262.

Foliaki, S, Best D, Akau'ola S, et al (2011). Cancer incidence in four pacific countries: Tonga, Fiji Islands, Cook Islands and Niue. Pac Health Dialog, 17, 21-32.

Foliaki S, Jeffreys M, Wright C, Blakey K, Pearce N (2004). Cancer in Pacific people in New Zealand: a descriptive study. Pac Health Dialog, 11, 94-100.

Jameson A, Sligo F, Comrie M (1999). Barriers to pacific women's use of cervical screening services. Aust $N Z J$ Public Health, 23, 89-92.

Lovell S, Kearns RA, Friesen W (2007). Sociocultural barriers to cervical screening in South Auckland, New Zealand. Soc Sci Med, 65, 138-50.

Ludeke, M, Puni R, Cook L, et al (2012). Access to general practice for Pacific peoples: a place for cultural competency. $J$ Prim Health Care, 4, 123-30.

Mehmetoglu HC, Sadikoglu G, Ozcakir A, Bilgel N (2010). Pap smear screening in the primary health care setting: A study from Turkey. $N$ Am J Med Sci, 2, 467-72.

Meredith I, Sarfati D, Ikeda T, Atkinson J, Blakely T (2012). High rates of endometrial cancer among Pacific women in New Zealand: the role of diabetes, physical inactivity, and obesity. Cancer Causes Control, 23, 875-85.

Minister of Health and Minister of Pacific Island Affairs (2010). Ala Mo'ui: pathways to pacific health and wellbeing 20102014. edited by Ministry of Health. Wellington

Obel J, Souares Y, Hoy D, et al (2014). A systematic review of cervical cancer incidence and mortality in the Pacific Region. Asian Pac J Cancer Prev, 15, 9433-7.

Sheridan NF, KenealyTW, Connolly MJ, et al (2011). Health equity in the New Zealand health care system: a national survey. Int J Equity Health, 10, 45.

Sligo F, Jameson A, Comrie M (1998). New Zealand polynesian women's access to information about cervical screening. $J$ Manag Med, 12, 361-9.

Taylor VM, Jackson JC, Schwartz SM, Tu SP, Thompson B (1996). Cervical cancer among Asian American women: a neglected publica health problem? Asian Am Pac Isl J Health, 4, 327-42. 